Revue européenne des sciences sociales

European Journal of Social Sciences

XLIV-134 | 2006

Quel(s) défi(s) pour les sciences sociales à l'heure de la mondialisation?

\title{
Introspection africaine et anthropologie : les paradoxes du libéralisme à la mozambicaine
}

\section{Severino Elias Ngoenha}

\section{(2) OpenEdition}

Journals

Édition électronique

URL : http://journals.openedition.org/ress/299

DOI : $10.4000 /$ ress.299

ISSN : 1663-4446

Éditeur

Librairie Droz

Édition imprimée

Date de publication : 1 juin 2006

Pagination : 193-202

ISBN : 9-782-600-01095-5

ISSN : 0048-8046

Référence électronique

Severino Elias Ngoenha, «Introspection africaine et anthropologie : les paradoxes du libéralisme à la mozambicaine », Revue européenne des sciences sociales [En ligne], XLIV-134 | 2006, mis en ligne le 14 octobre 2009, consulté le 21 avril 2019. URL : http://journals.openedition.org/ress/299 ; DOI : 10.4000/ress.299 
Severino Elias NGOENHA

\section{INTROSPECTION AFRICAINE ET ANTHROPOLOGIE: LES PARADOXES DU LIBÉRALISME A LA MOZAMBICAINE}

Le philosophe et journaliste guinéen Filomeno Lopes m'a suggéré d'introduire ma contribution par l'allégorie que j'avais utilisée lors d'un entretien avec lui et qu'il reprend avec une rigueur plus philosophique dans son ouvrage Filosofia intorno al fuoco. Il pensiero africano contemporaneo tra memoria e futuro ${ }^{1}$.

L'allégorie consistait à représenter le monde globalisé actuel comme un homme ayant épousé cinq femmes: la première s'appelle Afrique, la deuxième Asie, la troisième Europe, la quatrième Amérique et la cinquième Australie. L'Afrique est la première sans doute parce que, ayant vu naître le premier homme, elle est le berceau de l'humanité; ou peut-être parce qu'on y découvrit aussi le feu. Première épouse, l'Afrique est donc la plus âgée, la plus fatiguée, et par voie de conséquence la moins attrayante et la plus délaissée, voire abandonnée, après qu'une vie d'esclavage, la colonisation, la néo-colonisation et les nouvelles formes de paupérisation l'ont usée.

S'il est vrai que la situation de la vieille femme est propre à éveiller le chagrin et la compassion de témoins extérieurs, il est loin d'être certain que la situation actuelle de l'intéressée l'afflige autant que ces témoins. En effet, si on demandait à la première épouse du polygame une opinion sur son sort, elle nous dirait, peutêtre, qu'on a fini par la laisser «en paix »; que pour la toute première fois dans sa longue existence, elle a l'occasion de s'occuper d'elle-même, de se reposer un peu sans devoir satisfaire à tout prix les besoins bestiaux de son mari. Car qui peut nier que l'esclavage, le colonialisme, le néo-colonialisme, les guerres fratricides, les «guerres dites justes», les fabriques d'armes, les mines anti-personnel, la pédophilie, la prostitution systématique et les autres formes dantesques de l'actuelle culture de la mort sont des réponses à des besoins bestiaux?

Ce désir de l'épouse d'être «laissée tranquille», malgré l'injustice subie, montre que son «abandon» peut lui apparaître comme l'opportunité de penser à soi-même et d'être enfin maîtresse de son propre devenir historique.

Vue sous cet angle, la situation actuelle de l'Afrique ne serait pas nécessairement catastrophique. Le défi positif, porteur d'avenir, auquel la pensée et la philosophie africaines sont appelées à faire face serait une véritable metanoia consistant à transfigurer le cadre de l'expérience et à abandonner le pessimisme qui semble habiter nombre de nos substrats d'analyse actuelles - qui amènent par exemple Kä Mana à s'interroger ainsi: L'Afrique va-t-elle mourir?². Dans cette

\footnotetext{
Bologna, Editrice Missionaria Italiana, 2001, p. 170-171.
}

Paris, Karthala, 1993. 
perspective, il s'agirait de poursuivre une réflexion dont la logique, fondée sur la culture du dialogue et de la responsabilité, répondrait à l'exigence de faire de l'ordre dans notre propre maison. La situation d' «abandon» se révèle donc une occasion, pour la philosophie africaine en particulier, et la pensée africaine en général, de se confronter avec elles-mêmes.

Mais d'autres dangers guettent la vieille dame. Car il existe des gens prêts à tirer profit de la faiblesse, des individus sans feu ni lieu, ou peut-être malades, qui dominent et exploitent les faibles, enfants ou vieilles dames. C'est pourquoi si l'«abandon» peut être une occasion d'introspection et de mise en ordre de son propre espace existentiel, il constitue aussi un risque, éventuellement plus grand que le précédent, celui d'être à la merci des voyous du monde, sans «l'affection et la protection» du mari. Il s'agit donc, pour tirer parti de la situation actuelle, d'éviter de se «diluer dans l'universel sans pour autant se perdre dans l'isolement».

La vieille dame est l'âme africaine. Sa maison est le continent dans lequel Gérald Berthoud, cherchant à sortir de l'ethnocentrisme européen, a pratiqué le terrain, non comme l'ethnologue qui se contente de décrire des particularités, mais comme l'anthropologue pour qui l'étude monographique constitue une ouverture sur la diversité d'une condition humaine universelle. L'universel et le divers: deux dimensions qui s'allient dans la pensée de l'anthropologue comme dans l'image de la maison. C'est pourquoi, reprenant l'idée de notre ami commun Laurent Monnier, je parlerai ici du cadre du devenir africain comme de la maison Afrique. Parler de la maison Afrique signifie parler des parties qui composent la totalité de la maison. On peut partir de la totalité en direction des parties ou des spécificités des parties, ou d'une des parties, pour remonter en direction de la totalité de la maison.

Du point de vue génétique, je défends la primauté d'une idée générale d'Afrique par rapport à ces ensembles plus particuliers comme les Etats et d'autres configurations d'ordre régional. Les idées pan-négriste puis pan-africaine, nées dans les diasporas, ont précédé toute idée d'Etat. Toutefois, la perspective d'une Afrique fédérale mais unie (Nkrumah), d'une Afrique faite d'ensembles économiquement complémentaires (Mamadou Dia) ou d'ensembles culturellement homogènes (Cheikh Anta Diop), a cédé le pas face à la poussée néocoloniale de l'étrange entité qu'était et que reste l'espace de colonisation européen.

Malgré les réticences qu'on peut avoir par rapport à ce qu'on appelle normalement les Etats-nations africains, ils sont les seuls à être reconnus par les institutions internationales et, à ce titre, les acteurs politiques incontournables de ces cinquante dernières années. C'est pourquoi, si ordre il doit y avoir, il doit être pensé à partir de ses principaux tenants, qui sont aujourd'hui les Etats africains, et tirer parti des spécificités de chacun, pour les utiliser comme input, afin d'obtenir une vision d'ensemble de la maison Afrique.

La particularité à travers laquelle je vais essayer de m'approcher de la maison Afrique est le Mozambique. D'abord, parce que c'est mon pays et que j'en connais bien les vicissitudes politiques et historiques; mais surtout pour que soit évoqué, dans le cadre de la présente publication en hommage à Gérald Berthoud, le dernier terrain en date de sa carrière d'anthropologue ${ }^{3}$. Sans doute chaque partie

De ce travail, est né l'ouvrage A Longa Marcha duma «Educação para Todos» em Moçambique, en collaboration avec José P. Castiano et Severino E. Ngoenha, Maputo, Imprensa Universitária, 2005. 
de l'Afrique a une histoire et un parcours originaux, mais je soutiens que le Mozambique constitue une particularité plus particulière que d'autres, tout au moins que son histoire le rend atypique par rapport à la majorité des pays africains. Mais en même temps, il y a dans la trame historique mozambicaine des constantes qui peuvent servir à notre compréhension des problèmes qui se posent dans l'ensemble de la maison Afrique.

\section{QU'EST-CE QUI FAIT DONC LA PARTICULARITÉ DU MOZAMBIQUE?}

On pourrait citer d'abord le fait d'être devenu indépendant tardivement par rapport à la plupart des pays du continent; celui de faire partie de ces pays qui ont atteint leur indépendance après une guerre de libération; celui d'avoir connu une longue période de guerre dite civile, surtout aux premiers jours de l'indépendance et après la fin de la guerre de libération; celui d'avoir été capable de surmonter les conflits armés et de s'engager d'une manière exemplaire dans un processus de paix et de démocratie; celui enfin d'être passé d'un marxisme extrême à un libéralisme tout aussi extrême.

Mais c'est une autre caractéristique qui définit la spécificité du Mozambique: une présence permanente et quasi légale de la violence! On pourra me rétorquer: «En quoi la violence constante est-elle une spécificité du Mozambique?». Sur le fond, je n'aurais pas d'arguments convaincants, et serais donc obligé d'avouer que la violence est une constante dans la société africaine, non seulement à l'époque coloniale mais aussi dans la présente période post-coloniale. Pourtant, en termes de dosage, c'est-à-dire d'intensité, de durée, de forme, on peut défendre une spécificité de la violence mozambicaine.

La première institutionnalisation de la violence est liée à la nature dépendante du colonialisme portugais et plus profondément aux défaites et humiliations que le Portugal a subies de la part des autres puissances coloniales.

Dans la course pré-coloniale que le vocabulaire occidental a systématiquement appelée Ere des Grandes Découvertes, le Portugal avait été le premier à longer toute la côte africaine, à se faire propriétaire et à obtenir de la part de l'Eglise (Propaganda Fide et bulles papales) la légitimité de la colonisation au nom de la mission d'évangélisation. A la fin du XIX ${ }^{\mathrm{e}}$ siècle, le Portugal perd tout ceci. Les puissances coloniales occupent les terres qui appartiennent aux autochtones au nom du ius inventionis initié par Christophe Colomb, sanctionné juridiquement par l'école de Salamanque. La marginalisation du Portugal est telle à la fin du XIX ${ }^{e}$ siècle, qu'il n'est même pas appelé à participer au banquet des colonisateurs européens à Berlin en 1885. Quand il se rend compte que l'Eglise et la Propaganda Fide ont concédé ses possessions à d'autres Etats (comme par la reconnaissance du droit de la Belgique à coloniser le Congo), il proteste. Mais la Propaganda Fide répond que le Portugal s'est avéré incapable d'accomplir la mission qui lui avait été confiée en échange de l'occupation des terres (mission chrétienne); et ajoute que les autres Etats européens n'accepteront pas que le Portugal continue à être le détenteur légal de ces terres. Le Vatican reconnaît implicitement que les Etats-Nations européens sont les nouveaux sujets (fauteurs) de droit. 
L'échec et mat arriva en 1890 quand la Grande-Bretagne empêcha le Portugal, par un ultimatum, de se rabattre comme vers une bouée de sauvetage sur son dernier rêve colonial: relier le Mozambique à l'Angola par le train (carte rose); car ce projet entrait en concurrence avec l'ambition de Cecil Rhodes de relier le Cap au Caire.

Cette dernière humiliation provoqua un débat à Lisbonne à la fin du XIX siècle entre ceux qui pensaient que, suite aux défaites et humiliations successives, le Portugal devait renoncer définitivement à la colonisation et se concentrer sur l'Europe, et ceux qui pensaient que le meilleur moyen d'intégrer l'Europe et d'y être reconnu était de posséder des colonies rentables.

Ce deuxième courant - constitué par de jeunes journalistes comme Luciano Cordeiro $^{4}$ et António Enes ${ }^{5}$, membres de l'Institut de Géographie récemment créé - prévaudra. Il est à l'origine des lois sur le travail forcé. L'esclavage, qui venait à peine d'être aboli dans les colonies portugaises, fut remplacé par la légalisation du travail forcé, promulguée en 1914, qui restera en vigueur jusqu'aux années soixante. Avec cette loi, le colonisateur portugais disait: «Nous avons la terre, le nègre pour travailler, la police pour réprimer et encadrer, et des administrateurs pour contrôler. Ce dont nous avons besoin, ce sont les investissements ». Le Portugal reconnaissait ainsi la nature dépendante et répressive de son colonialisme.

A regarder de près le fonctionnement politique et social du régime démocratique du Mozambique libéral d'aujourd'hui - et nous avons là une constante applicable à la quasi-totalité des régimes politiques africains -, on peut se demander s'il ne comporte pas implicitement le même postulat, à savoir: "Nous avons la terre, la police, les administrateurs, les nègres, ce qui nous fait défaut ce sont les investissements ». Ce faisant, nos régimes politiques n'avouent-ils pas, comme jadis le colonisateur portugais, leur nature dépendante et violente?

Le Portugal d'Enes et de Cordeiro avouait vouloir utiliser la colonisation comme moyen pour son intégration à l'Europe, seul monde qui lui semblait attrayant. Et nous, par nos agissements politiques, quelle maison voulons-nous intégrer? Ne nous tiendrions-nous pas, comme nos anciens exploiteurs, dans le vestibule de l'Europe, prêts à abandonner la maison Afrique?

L'historien portugais António da Rita Ferreira disait du Mozambique qu'il était une colonie britannique sous administration portugaise. Suite aux événements de la fin de la guerre froide et aux difficultés politiques et économiques que connaissent nos pays, suite aux programmes endémiques d'ajustement structurel ou, si nous voulons, à la présence toujours accrue des institutions internationales dans la définition des politiques gouvernementales, au Mozambique mais aussi dans les différents pays du continent, ne sommes-nous pas en train d'abdiquer, de fait, notre indépendance et notre droit à l'autodétermination, pour nous confiner dans un rôle de simples administrateurs des intérêts des dominants?

La nature fasciste du régime colonial portugais et son anachronisme historique nous ont poussés à faire recours aux armes comme seul moyen de nous opposer

\footnotetext{
${ }^{4}$ Questões coloniais, Lisboa, Editorial Vega, 1980.

5 Moçambique - Relatorio Apresentado ao Governo, Lisboa, Agência Geral do Ultramar, 1971.
} 
à la violence et d'atteindre à l'indépendance politique; cela, comme l'a relevé A. Cabral, sans jamais confondre le colonialisme avec le peuple portugais ou la race blanche. Nous avons fait une guerre de libération «juste» et nous l'avons gagnée. Peut-être pas militairement, mais ce n'est pas cela qui nous intéressait; la bataille décisive était politique, et celle-là fut gagnée. En juin 1975, nous sommes devenus un pays indépendant. Avec un retard d'une décennie, nous avons, enfin, rejoint la maison Afrique indépendante.

Malheureusement, à la violence coloniale s'est substituée la violence de l'idéologie marxiste - autre constante historique. En effet, la quasi-totalité de la maison Afrique s'est contentée de remplacer la violence coloniale par de nouvelles violences idéologiques au nom de la gauche, de la droite ou du socialisme africain. Et comme si cela ne suffisait pas, peu de temps après, nous avons été entraînés - par nos contradictions internes, par la situation internationale mais surtout à cause de ceux qui ne voulaient pas nous voir libres, comme disait une poésie de Nyerere - dans une deuxième guerre, dite civile ${ }^{6}$. Guerre beaucoup moins héroïque que la précédente, menée avec des «intentions étranges et pour des raisons inavouables »; beaucoup plus meurtrière et destructrice que la première aussi; d'une violence effrénée et souvent gratuite, qui a tragiquement aggravé les effets de la famine et de la misère.

A qui a servi cette guerre? Qu'est-ce que le pays a gagné? Qu'est-ce que le pays a perdu? Qui sont les (vrais) vainqueurs? - Cette série de questions ressortit à cette constante historique des conflits qui traversent le continent (la maison Afrique) depuis les indépendances. Quelles sont les raisons de ces conflits? A qui servent-ils? Quelles sont leurs conséquences pour les populations africaines? Je vais essayer de répondre à ces questions pour le Mozambique (et quelques réponses s'appliquent peut-être à d'autres situations) au travers de trois analyses possibles de la situation historique: soit trois différents scénarios.

\section{Premier scénario : le pays a perdu}

\section{L'économiste mozambicain António Maganza soutient que}

... la guerre civile a signifié pour notre pays un recul du point de vue d'abord humain
(nous avons détruit tout le tissu social que la première République et même, avant elle, la
période de libération, avaient tramé à grand-peine; aujourd'hui nous sommes des triba-
listes, racistes, mais surtout nous avons contracté cette nouvelle maladie occidentale
qu'on appelle l'individualisme), mais aussi économique (le peu d'infrastructures dont on
disposait a été méticuleusement détruit et notre dette extérieure est une des plus impor-
tantes au monde).

On peut défendre avec une relative facilité la thèse d'une défaite du pays dans le deuxième conflit. En effet, le nombre de morts de toutes les zones du pays a été terriblement élevé. Le pays a été complètement détruit. La guerre s'est orientée vers la destruction des quelques infrastructures qui maintenaient le pays debout. A tel point que l'obstination à atteindre systématiquement les infrastructures écono-

- Severino E. Ngoenha, Por uma dimensão moçambicana da consciência histórica, Porto, Ed. Salesianas, 1992.

Hebdomadaire Savana, Maputo-Mozambique, février 1997. 
miques, avec une opiniâtreté et une efficience je ne dis pas admirables, mais scandaleuses, porte à soupçonner l'intervention de forces extérieures, visiblement animées d'intentions destructrices. Comme si cela ne suffisait pas, à la fin de la guerre, la dette extérieure du pays avait augmenté de manière exponentielle. Du point de vue social, tous les réseaux de solidarité ont été détruits. A leur place est née la méfiance entre voisins, entre familles et même entre membres de la même famille.

Le Mozambique connaît aujourd'hui une préoccupante vague de tribalisme et de régionalisme, qui découle directement de cette deuxième guerre. Ce phénomène constitue un sérieux recul par rapport à la première République (19751990), qui avait fait de la lutte contre le tribalisme un des points forts de la construction de la mozambicanité; bien plus, ce tribalisme/régionalisme constitue un recul par rapport à la politique assimilationniste du Portugal, qui obligeait les identités locales à se situer relativement à la métropole, empêchant tout élan tribaliste ou régionaliste. Le dernier mais non le moindre de ces facteurs de déstructuration sociale est un racisme qui fait son grand retour.

$\mathrm{Au}$ fond, toutes les valeurs humaines qui caractérisaient la société mozambicaine, surtout sous la première République, semblent avoir disparu pour faire place à la «dollarocratie». Les relations humaines, en dépit de toute la dimension maussienne du don, et même contre la dimension holistique de l'appartenance à un groupe, cèdent face aux exigences de l'intérêt et du calcul, connotations spécifiques de l'individualisme. Comment, dans une société où la seule chose qui compte est ce seigneur extraordinairement détestable appelé Je, parler de maison, en donnant à ce terme un sens de solidarité, fraternité, convivialité, partage?

On peut étendre ce jugement à la quasi-totalité des guerres africaines, à propos soit du recul de tous les systèmes ou réseaux humains et sociaux ou communautaires, soit de la destruction du peu d'infrastructures existantes, soit encore de l'augmentation de la dette extérieure.

\section{Deuxième scénario: le pays (peuple) a gagné}

Le politicien de la RENAMO, Raúl Domingos, affirme de manière péremptoire, dans le journal Demos: «Nous avons enfin atteint la véritable liberté. Il s'ouvre maintenant pour le pays la possibilité d'une véritable convivialité civile et la possibilité d'entamer un véritable processus de développement. Ceci est dû à la guerre de la RENAMO.» ${ }^{8}$

Il n'est pas rare d'entendre défendre l'idée d'une soi-disant victoire du pays. En premier lieu, on avance l'argument selon lequel la première et principale victoire du peuple et du pays est la paix ${ }^{9}$. Soit! Mais qu'est-ce que la paix? Est-ce une simple absence de guerre? Le concept de paix, comme il est utilisé, implique un processus de réconciliation pour pouvoir être, sinon perpétuelle comme disait Kant, du moins durable. Cette paix suppose une plus grande participation, du plus grande nombre, à la vie collective.

s Hebdomadaire Demos, Maputo-Mozambique, février 1999.

Roberto Morozzo della Rocca, Mozambico. Una pace per l' Africa, Milano, Leonardo International, 2002. 
Mais d'une manière plus fondamentale, la question se pose de savoir quelle est la relation entre paix et justice. Un système politique injuste ou perçu comme tel par la majorité de la population est nécessairement source de conflits. En effet, il existe une relation intrinsèque entre l'injustice (ou réalité perçue comme injuste) et la violence. La deuxième République est perçue par la population comme individualiste et injuste. Il serait utile d'introduire l'idée du muséologue Jacques Debri, selon laquelle les portes - qui sont dans le monde beaucoup plus nombreuses que les hommes - servent à fermer et non à ouvrir. Plus une société est inégalitaire, injuste et violente, plus elle a besoin de portes.

Depuis le début de la deuxième République, on n'a pas seulement augmenté le nombre de portes dans la zone chic de Maputo et dans les autres grandes villes, mais on y a ajouté des grilles, chiens de garde, alarmes, gardiens armés, agents de police, afin d'empêcher que les damnés ne viennent déranger la tranquillité des seigneurs. Simultanément, le nombre d'enfants de la rue a augmenté, le nombre de fillettes prostituées également, ainsi que le nombre de voleurs, de bandits, c'est-à-dire de pauvres.

Le Mozambique libéral a vu augmenter le nombre des banques, et même le private banking sur tout son territoire; les villas chic se sont mises à pousser comme des champignons, les voyages à Nelspruit, Lisbonne et Dubaï pour le shopping, à Rio de Janeiro pour le carnaval, se sont multipliés; simultanément, le pays a été reçu au programme IPIC de la Banque Mondiale destiné aux plus pauvres du monde.

Le deuxième argument, normalement invoqué pour prouver la victoire du peuple dans la deuxième République, est l'instauration de la démocratie. Comment nier qu'il s'agit là d'une victoire pour le peuple? De fait, le Mozambique d'aujourd'hui est beaucoup plus démocratique que celui d'hier. Je puis énumérer plusieurs indicateurs, à commencer par la nouvelle Constitution, l'existence d'un Parlement qui se veut représentatif et qui tente de fonctionner, l'existence de partis politiques légaux, de moyens de communication privée (radios, télévision, journaux indépendants), d'un enseignement secondaire et supérieur privé, et d'associations, forums de débat, etc.

Tout cela est fort appréciable. Toutefois, je ne puis me défendre contre un léger doute sur la nature de la démocratie mozambicaine - et des démocraties africaines - et la capacité des partis politiques à être représentatifs sous leur forme actuelle.

J'ai aussi des difficultés avec la capacité des populations africaines (mozambicaine en particulier) à comprendre et participer d'une manière consciente à une démocratie qui ne parle pas le langage des gens, qui ne puise pas dans l'imaginaire collectif des populations, mais se présente comme une copie des institutions politiques et juridiques de l'Occident. Celles-ci constituent des solutions aux problèmes auxquels les Européens ont été confrontés à un moment de leur histoire, mais menacent de n'être pour nous qu'un problème supplémentaire, plutôt qu'une solution, si elles ne sont pas repensées, acculturées de manière critique. Du point de vue philosophico-politique, si le peuple n'a pas la capacité de suivre les débats politiques, il ne peut sérieusement donner un mandat aux gouvernants, aux députés: par conséquent il n'est pas en mesure de légitimer la démocratie mozambicaine.

Si la démocratie ne tire pas sa légitimité de l'intérieur du pays et du peuple, elle doit la chercher à l'extérieur; mais où? Auprès de la communauté ou des 
organisations internationales? des ambassades? des fondations Carter ou Ford? Ces institutions peuvent-elles s'autoriser, politiquement et moralement, du pouvoir de légitimer et de garantir les démocraties africaines?

Troisième argument: le marché libre. Théoriquement, chaque Mozambicain peut devenir propriétaire et investir librement. Mais, si nous faisons l'histoire du Mozambique depuis le XIX ${ }^{\mathrm{e}}$ siècle, nous nous rendons compte que les Mozambicains n'ont jamais eu les moyens économiques pour investir. Quand on dit aujourd'hui que tous peuvent investir, en sachant que, jusqu'en 1990, personne n'avait rien parce que tout était supposé appartenir à tous, on peut se demander d'où est censée venir la manne qui fera de tous les Mozambicains des investisseurs.

D'où tient-on que les Mozambicains, qui n'ont jamais été propriétaires de quoi que ce soit, vont apprendre du jour au lendemain à être gestionnaires, patrons, managers? Ce dogme néo-libéral (qui a évacué l'exigence smithienne du respect des règles étatiques et même divines) ne contient-il pas en soi les germes de la corruption, et par conséquent de la violence à laquelle on assiste au Mozambique, j'ose même dire dans toute l'Afrique? Cela n'a-t-il pas mené à une confusion entre le secteur public et le secteur privé, entre l'homme politique et l'entrepreneur? Ou pis, la politique n'est-elle pas devenue un moyen pour atteindre des objectifs individuels de caractère économique? Par ce biais, ne falsifie-t-on pas la démocratie et le débat politique?

Comme l'ont soutenu plusieurs sociologues, le risque est de devoir hypothéquer la liberté des peuples au profit des alliances possibles entre les élites (politico-économiques, sans exclure la participation des élites intellectuelles), et les intérêts de certaines factions économiques du Nord ultra-libéral.

\section{Troisième scénario : les vainqueurs ne sont pas ceux qu'on croit}

Emidio Simango, juriste mozambicain, soutient qu'il y a bien de véritables vainqueurs de la guerre civile du Mozambique, mais qu'il ne faut pas les chercher à Maputo, mais au nord du monde où est en cours un nouveau partage de l'Afrique ${ }^{10}$.

Jusqu'il y a peu, les douanes mozambicaines étaient confiées à une entreprise britannique. Il est impressionnant d'arriver à Maputo et de tomber sur un Britannique à l'accent reconnaissable entre tous, qui vous enjoint d'ouvrir vos valises ! Le Portugal contrôle l'essentiel des transactions financières du pays au travers du quasi-monopole exercé sur le système bancaire. La France a obtenu le contrôle des frontières maritimes, les EUA veulent exploiter la très riche vallée du Zambèze, s'approprier le barrage de Cabora Bassa (le plus grand barrage de l'Afrique après Assouan), et installer une base militaire au nord du pays (Nacala). De nombreuses entreprises à la déontologie douteuse - liées à la drogue, aux produits toxiques, à la pédophilie, à la prostitution, au piratage de la bio-diversité, etc. - ont trouvé dans le faible Mozambique d'aujourd'hui un terrain favorable pour mener leurs affaires. De nombreux Portugais, qui avaient quitté le Mozambique au moment de la transition politique vers l'indépendance (par fuite ou par expulsion), reviennent avec une énorme soif de vengeance et tentent de se

${ }^{10}$ A nova partilha de Africa. Lisboa, Ed. Escritores 1992. 
réapproprier des biens immobiliers et de petites entreprises qui ont symbolisé, ces vingt-cinq dernières années, nos indépendances et nos libertés retrouvées. Une grande partie des élites qui étaient devenues nos prétendues classes moyennes, et s'étaient installées dans ce qu'on appelait la cité blanche, ont graduellement été repoussées en direction de la zone sub-urbaine, comme pour signifier que le temps des vacances (indépendances) était révolu.

Notre puissant voisin, la République Sud-Africaine (démocratique et gouvernée par des Noirs de l'ANC, pour laquelle beaucoup de Mozambicains ont donné leur vie, et le pays consenti des sacrifices économiques et militaires énormes) aujourd'hui humilie, maltraite, expulse les Mozambicains de son territoire, mais surtout mène une politique économique fort dommageable au peu de capacité productive qui subsiste au Mozambique. En effet, en inondant littéralement le petit marché mozambicain de ses produits, l'Afrique du Sud d'aujourd'hui pousse notre dépendance à un point que nous n'avons pas connu, même quand les maîtres du jeu étaient les Boers à Pretoria et des colonialistes à ce qu'on appelait alors Lourenço Marques...

J'ai envie de dire, d'une manière très samorienne: «A luta continua!». Il s'agit d'une lutte contre la violence (militaire, politique mais aussi sociale) et ses causes, pour aboutir enfin à la paix. Dans cette lutte ou, si l'on veut, dans cette réorganisation de la maison Afrique, deux perspectives me semblent importantes: l'une, dans nos rapports avec le monde extérieur, l'autre, dans notre réorganisation politique et sociale interne.

Pour ce qui est de la première perspective, on peut s'inspirer de la thèse fondamentale d'Ulrich Beck dans la Société du risque ${ }^{11}$, selon laquelle l'Occident produit, comme jamais auparavant, une quantité impressionnante de biens, dont le corollaire est la production de risques. D'après Beck, la seule chose que l'Occident est prêt à partager et qu'il exporte donc vers l'Afrique, ce seraient les risques. Ce qu'on appelle globalisation serait une globalisation des risques. Si on utilise ce raccourci, on peut penser que les grands défis auxquels nous sommes aujourd'hui confrontés par rapport au monde global ou globalisateur serait de créer une stratégie visant à réduire l'importation des risques et en même temps augmenter notre capacité à importer des biens. Le rôle des scientifiques dans cette entreprise est fondamental, car la discrimination entre ce qui est risque et ce qui est bien ne se fait pas à l'œil nu, mais relève de la connaissance scientifique.

Pour ce qui est du problème des relations internes de la maison Afrique, sa solution me paraît passer par l'élaboration d'un véritable pacte africain qu'il faut, je crois, considérer sous trois aspects.

Premièrement, il me semble nécessaire de conclure ou de renouveler un véritable contrat social ${ }^{12}$ entre les diverses parties de la société, et tout d'abord entre les élites et la population. Il est nécessaire que les gens aient confiance en ceux

${ }^{11}$ U. Beck, La Société du risque: sur la voie d'une autre modernité, Paris, Flammarion, 2003 (trad. fr. de Risikogesellschaft: auf dem Weg in eine andere Moderne, Frankfurt a.M., Suhrkamp, 1986).

12 Severino E. Ngoenha, «Identidade moçambicana: já e ainda não», in Carlos Serra (dir.), Identidade, moçambicanidade, moçambicanização, Maputo, Livreria Universitáris, Universidade Eduardo Mondlane, 1998, pp. 17-34. 
que leurs compétences appellent à diriger le pays. Pour cela, les «talented tenth» dont parlait Dubois, les gens doués, doivent revenir à l'Afrique, s'y retrouver pour y être reconnus, et ainsi contribuer à l'épanouissement de leur propre univers culturel. Cela ne concerne pas que les élites politiques et économiques, mais bien aussi intellectuelles, au sens où les détenteurs d'un capital culturel ${ }^{13}$, d'origine occidentale, ont à repenser (convertir?) la valeur de ce capital au sein de la maison Afrique, orienter leurs travaux, leurs recherches, en fonction de ses besoins, poser à leurs savoirs les questions comme elles se posent dans ce continent en mutation que son intelligence fuit, ou ignore trop souvent.

En outre, je crois qu'il n'est pas moins nécessaire, pour les élites intellectuelles, de proposer à la société africaine une sorte de nouveau contrat culturel $^{14}$ conçu comme une redéfinition de l'identité africaine. Cela veut dire ancrer le droit et les institutions politiques et sociales dans les coutumes et les structures sociales des populations locales, puiser dans l'imaginaire africain et s'y enraciner. La culture d'Etat doit apprendre à parler le langage des gens, c'est-à-dire qu'il nous faut, d'une part, assumer l'héritage culturel laissé par l'Occident et, d'autre part, s'approprier cet héritage, l'acculturer de façon critique dans ce que Sartre a appelé la «négritude objective». N'est-ce pas là du reste la mission de toute anthropologie véritable?

Enfin, la nécessité d'un contrat politique ${ }^{15}$, d'une domestication des conflits, apparaît criante devant la violence belliqueuse chronique qui infeste le continent depuis un demi-siècle. Il faut que les différents acteurs de la scène politique (non seulement les chefs et leur base, mais toutes les parties en présence, jusqu'aux courants marginaux) entrent dans une dynamique commune de gestion des conflits et apprennent à devenir partenaires, tout au plus adversaires, mais jamais ennemis mortels.

Que ceux qui détiennent la force apprennent à parler, et à se parler, sans intermédiaires, qu'ils montrent leur vaillance à exceller dans l'art subtil de la palabre, espace de confrontation où l'on se bat par l'écoute, le dialogue, la compréhension; où la victoire s'appelle pardon; où le sens d'une appartenance commune ferme la porte aux négociateurs extérieurs, et autres influences occultes. Cela aurait comme avantage majeur de nous obliger à trouver nous-mêmes des solutions à nos propres problèmes. Plaidons donc pour que la maison Afrique se (re)constitue comme famille et assume dans ses propres domaines ce que «la liberté implique comme responsabilité ${ }^{16}$.

Institut d' anthropologie et de sociologie

Université de Lausanne

Severino.Ngoenha@unil.ch

${ }^{13}$ Gérald Berthoud appréciera sans doute le choix de l'expression.

${ }^{14}$ Severino E. Ngoenha, Por uma reconciliação entre a poítica e a(s) cultura(s), Maputo, José Elija M. Guambe et Bernhard Weimer (éds.), 1997.

${ }^{15}$ Severino E. Ngoenha, As rações da reforma constitutional, J. E. M. Guambe et B. Weimer (éds), Maputo, Ministerio de administração estatal, 1998.

${ }^{16}$ Booker T. Washington, L'autobiographie d'un noir, Paris, Plon, 1961 (trad. de l'anglais). 